\title{
Commentary on 'Evaluating tissue-engineered repair material for pelvic floor dysfunction: a comparison of in vivo response to meshes implanted in rats'
}

\author{
Sundas Tahir Masudi ${ }^{1} \cdot$ Abdelmageed Abdelrahman $^{2}$ (I)
}

Received: 7 February 2022 / Accepted: 9 February 2022 / Published online: 8 March 2022

(c) The International Urogynecological Association 2022

The aim of this study was to investigate host response to the tissue-engineered repair material (TERM) of adiposederived stem cells (ADSCs)-polyglycolide (PGA) terpolymers (PLTG) in rat abdominal defect models through comparing different materials and to study the changes in biomechanical properties over time. In doing so, better anatomic restoration and a decreased incidence of associated complications may be achieved, a most favourable outcome in the material repair of pelvic floor dysfunction (PVD).

ADSCs were seeded on electrospun PLTG terpolymers in six-well plates to construct the TERM. The TERM, PLTG, porcine small intestine submucosa mesh (SIS), and polypropylene (PP) ( $n=6 /$ group per time point) were implanted in rats for 7, 30, 60 and 90 days. Hematoxylin-eosin and Masson's trichrome staining were used to assess the host response and mechanical testing was used to evaluate the changes in biomechanical properties. The results of this study demonstrated that the ADSCs was confined to the abdominal wall and that the TERM was encapsulated by a thicker layer of connective tissue. The TERM was also associated with less reduced inflammatory scores compared with PLTG and PP over time and its vascularisation was greater than that of PP and PLTG over time $(p<0.05)$ and also greater than that of SIS on days 90 . The ultimate tensile strain and Young's modulus of the PP group showed the greatest increases and the TERM group followed on day 90 .

This study concluded that tissue-engineered material of ADSCs-PLTG increased the encapsulation with host tissue and also reduced inflammation in rats when compared to PP and PLTG. The biomechanical properties of this TERM were found to be less than those of PP and greater than those of SIS on day 90. Therefore, the TERM of ADSCs-PLTG may be an alternative material for the repair of POP and SUI. However, there are a number of limitations to this study including the possibility that the ADSCs-PLTG may not meet the mechanical requirements of pelvic floor repair. Furthermore, abdominal wall tissue is different from vaginal tissue and the relatively short duration of the study meant that biomechanical properties and histopathologic outcomes could not be evaluated in the longer term. Future long-term studies involving larger animals are required to evaluate TERM for pelvic floor dysfunction.

Publisher's note Springer Nature remains neutral with regard to jurisdictional claims in published maps and institutional affiliations.
Abdelmageed Abdelrahman

abdelmageed@hotmail.co.uk

School of Medicine, University of Liverpool, Liverpool, UK

2 Department of Urogynaecology, Liverpool Women's Hospital NHS Foundation Trust, Liverpool, UK 